
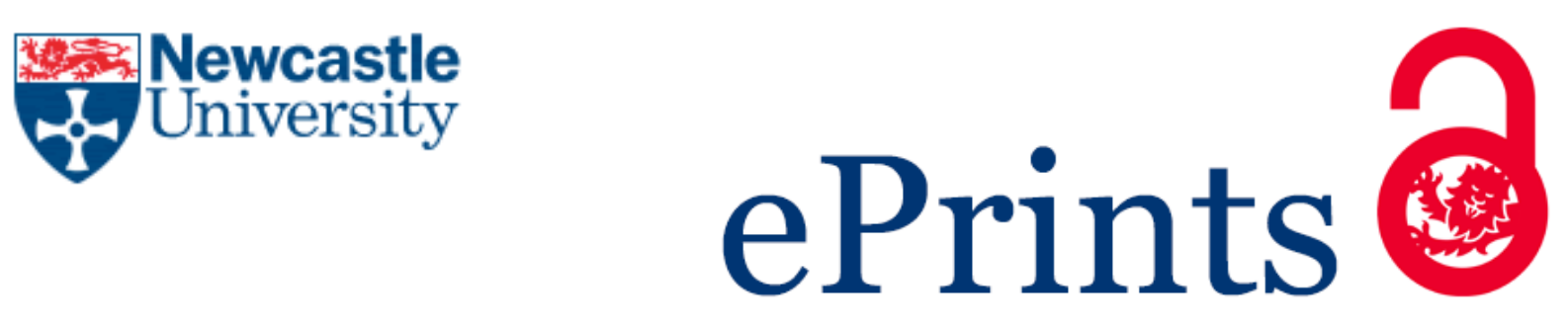

Ladha C, Del Din S, Nazarpour K, Hickey A, Morris R, Catt M, Rochester L, Godfrey A.

Toward a low-cost gait analysis system for clinical and free-living assessment.

In: 38th Annual International Conference of the IEEE Engineering in Medicine and Biology Society (EMBC).

16-20 August 2016, Orlando, Florida: IEEE.

(C) 2016 IEEE. Personal use of this material is permitted. Permission from IEEE must be obtained for all other uses, in any current or future media, including reprinting/republishing this material for advertising or promotional purposes, creating new collective works, for resale or redistribution to servers or lists, or reuse of any copyrighted component of this work in other works.

Conference website:

http://embc.embs.org/2016/

Date deposited:

$22 / 06 / 2016$ 


\title{
Toward a low-cost gait analysis system for clinical and free-living assessment
}

\author{
Cassim Ladha, Silvia Del Din, Member, IEEE, Kianoush Nazarpour, Senior Member, IEEE, Aodhán \\ Hickey, Rosie Morris, Michael Catt, Lynn Rochester, and Alan Godfrey, Member, IEEE
}

\begin{abstract}
Gait is an important clinical assessment tool since changes in gait may reflect changes in general health. Measurement of gait is a complex process which has been restricted to bespoke clinical facilities until recently. The use of inexpensive wearable technologies is an attractive alternative and offers the potential to assess gait in any environment. In this paper we present the development of a low cost analysis gait system built using entirely open source components. The system is used to capture spatio-temporal gait characteristics derived from an existing conceptual model, sensitive to ageing and neurodegenerative pathology (e.g. Parkinson's disease). We demonstrate the system is suitable for use in a clinical unit and will lead to pragmatic use in a free-living (home) environment. The system consists of a wearable (tri-axial accelerometer and gyroscope) with a Raspberry Pi module for data storage and analysis. This forms ongoing work to develop gait as a low cost diagnostic in modern healthcare.
\end{abstract}

Presented at the $38^{\text {th }}$ Annual International Conference of the IEEE Engineering in Medicine and Biology Society, Orlando, Florida, USA

Conference: http://embc.embs.org/2016/

16-20 August 2016

Available on IEEEXplore.

\section{Introduction}

Human locomotion or gait is a useful measure of overall health [1], cognitive decline [2], falls status [3] and longevity [4]. Therefore, measuring characteristics of gait is important as a robust method to determine many facets of health [5]. Traditionally, spatio-temporal characteristics of gait have only been quantifiable in bespoke clinical/research facilities with large and expensive equipment, e.g. instrumented walkways or 3D motion analysis. However, with the advent of wearable technology for pervasive health monitoring the influx of inertial measurement units incorporating accelerometers and gyroscopes has seen their use flourish due to the reduced cost, unrestricted use, continuous monitoring (i.e. not limited to set distance or location) and translation to various physical capability tasks other than gait [6-8]. The utility of such a discrete and multifunctional tool affords accurate and objective monitoring to extend beyond the clinic/laboratory and into the wearers home or in the general community for older adult and/or pathological (e.g. Parkinson's disease) research [9]. While validated commercial wearable gait systems exist, they remain expensive, use patented or proprietary software and closed source implementations of unpublished work. There is a need for an open source gait system and processing pipeline to facilitate pragmatic and transparent use.

In this pilot work, commercially available technology is used to show the feasibility of assembling a low-cost, clinically relevant gait analysis system with previously validated methodologies. The system comprises entirely open source components and is part of our ongoing work to implement gait as a low-cost diagnostic in modern healthcare.

\section{Related work}

\section{A. A conceptual model of gait}

Previous work has outlined a clinically defined conceptual model of gait, sensitivity to ageing and pathology $[1,2,10]$. The model presents 16 spatio-temporal (micro) gait characteristics [1, 10], 14 quantifiable by a single tri-axial accelerometer wearable worn on the lower back (fifth lumbar vertebrae, L5) [9]. Additionally, free-living behavioural (macro) gait characteristics can be quantified from the same wearable by examining the broader trends of the same signal [10]. Therefore, outcomes relating to total time spent walking, number of steps, accumulation of time walking and adherence to health guidelines may also be quantified [11].

Research supported by the Engineering and Physical Sciences Research Council (EPSRC), EP/N508718/1. AG and LR are supported by the National Institute for Health Research (NIHR) Newcastle Biomedical Research Centre (BRC) and Unit (BRU) at Newcastle upon Tyne Hospitals NHS Foundation Trust and Newcastle University. Work also supported by the NIHR Newcastle Clinical Research Faculty infrastructure funding. Views are those of the authors.

C. Ladha, S. Del Din, A. Hickey, R. Morris, M. Catt, L. Rochester and A. Godfrey* are with the Institute of Neuroscience | Newcastle University Institute of Ageing, Newcastle University, NE4 5PL, UK. (*phone: +44-191-2081245; fax: +44-191-2081251; e-mail: cassim.ladha@ncl.ac.uk, silvia.deldin@ncl.ac.uk, rosie.morris@ncl.ac.uk, aodhan.hickey@ncl.ac.uk, michael.catt@ncl.ac.uk, lynn.rochester@ncl.ac.uk, alan.godfrey@ncl.ac.uk

K. Nazarpour is with the School of Electrical and Electronic Engineering | Institute of Neuroscience, Newcastle University, NE1 7RU, UK (kianoush.nazarpour@ncl.ac.uk). 


\section{B. Micro gait algorithms}

Two algorithms $[12,13]$ have been previously validated to quantify spatio-temporal gait characteristics with a wearable on L5 [9, 14]. In short, identification of the initial (IC) and final contacts (FC) events within the gait cycle are required to estimate temporal characteristics (e.g. step time) as well as determine the spatial characteristic (e.g. step length) within the inverted pendulum model of the gait cycle [13]. However, the spatial characteristic is dependent on a known variable, i.e. leg length or height of wearable from the ground. A combination of step time and step length estimates step velocity [9]. Once the spatiotemporal characteristics are known, variability (step fluctuations) and asymmetry can be examined from left and right steps, (1) and (2).

$$
\begin{aligned}
& \text { Variability }_{\text {Left \& Right }}=\sqrt{\frac{\text { variance }_{\text {left }}+\text { variance }_{\text {right }}}{2}} \\
& \text { Asymmetry }_{\text {Left \& Right }}=\mid \text { average }_{\text {left }}-\text { average }_{\text {right }} \mid
\end{aligned}
$$

The algorithms have been implemented and validated using MATLAB ${ }^{\circledR}$. However, wide-scale development and extension of MATLAB ${ }^{\circledR}$ based algorithms is limited due to licensing restrictions and cost. For this pilot development work an open source scripting language is adopted to calculate micro gait outcomes from the wearable data.

\section{Macro gait algorithms}

Utilising the same accelerometer signals but over prolonged periods (7 days) one can examine the general trends of the signal to identify walking periods (bouts) to examine total time walking, length of each bout or distributions [10]. Bouts have been examined and shown to have log normal distributions resulting in outcomes such as the power law scaling component $(\alpha)$ or the Gini index $(G)$ relating to statistical dispersion [15]. Identification of bouts can be estimated by examining (i) the mean and standard deviations [16] (ii) orientation and (iii) identification of IC/FC events [12] within a possible bout of walking. Algorithms $[11,16]$ and outcomes have been generated and validated using MATLAB ${ }^{\circledR}$ and thus have deploy-ability issues previously outlined. (Quantification of macro gait outcomes with the proposed system will be presented in future work).

\section{System design}

To ensure complete system utility across a range of testing environments and usability in various habitual scenarios a modular design was adopted, Fig. 1.

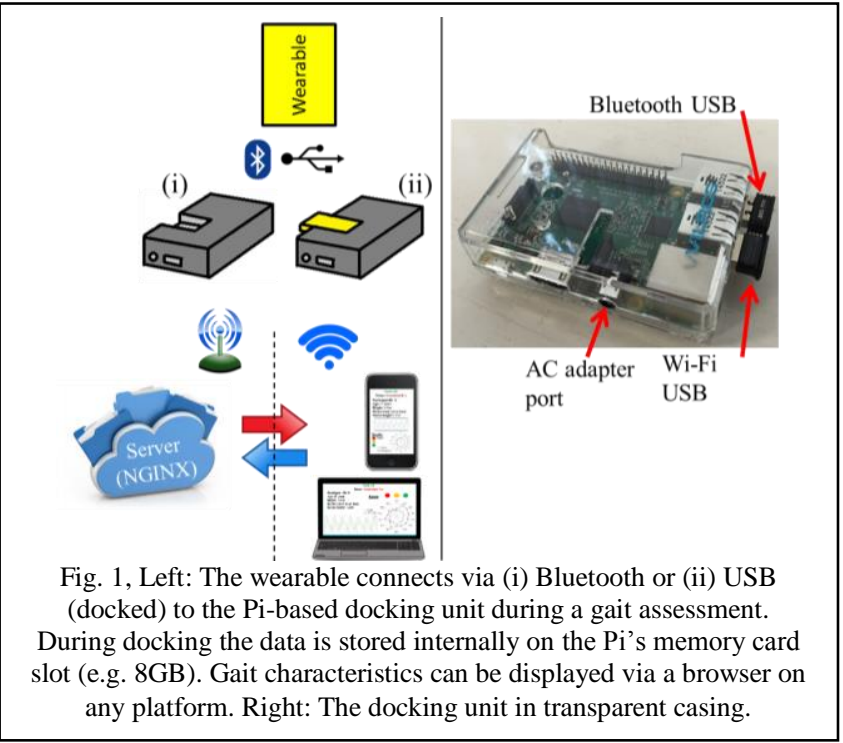

This pilot work is conducted in a supervised gait assessment condition (clinic). Here we capture data from two wearables and present three comparisons between the proposed system, streamed data and a validated reference:

\section{Equipment - proposed system}

Wearable: In the current design a single wearable with a tri-axial accelerometer $(50 \mathrm{~Hz}, \pm 8 \mathrm{~g}, 14 \mathrm{bit})$ and gyroscope $(50 \mathrm{~Hz}$, $2000^{\circ} / \mathrm{s}, 16$ bit) was used (WAX9, Axivity, York, UK: $23.0 \times 32.5 \times 7.6 \mathrm{~mm}$ ). The wearable is of an open source design [17] and was worn on L5 with the use of double sided tape and an additional bandage (Hypafix, BSN Medical Limited, UK). The wearable was configured and charged through a USB interface and data transmitted to a receiving docking unit.

Docking unit: This $(8.7 \times 6.0 \times 2.7 \mathrm{~cm})$ consisted of a Raspberry Pi 2 (Model B, 1GB RAM with 8GB micro SD card). Data were received by a Bluetooth adapter (Asus USB-BT $4003 \mathrm{Mbps}$ v4.1) and processed post walking by a Python ${ }^{\mathrm{TM}}$ script $^{\mathrm{i}}$ translated from a MATLAB ${ }^{\circledR}$ based algorithm as previously described [9]. The purpose of the docking unit is to provide unsupervised and continuous gait monitoring. Though gait data could be relayed to a researcher's smart phone, if protocols required longitudinal assessment (e.g. hours, days) the unit can collect, store or transmit data accordingly (please see Discussion, Testing scenario C). 
The Python $^{\mathrm{TM}}$ analysis made use of several open source libraries that offer similar functionality to their MATLAB ${ }^{\circledR}$ counterparts, namely: matplotlib; SciPy; NumPy; and pandas. Python ${ }^{\mathrm{TM}}$ was primarily chosen due to its platform portability and its native inclusion on POSIX based operating systems (supported on modern phones, tablets and web servers). Upon completion of walking the raw data was processed on board the Pi and results (14 spatio-temporal gait characteristics) were uploaded to an online server (configured using open source NGINX platform) via the Wi-Fi Nano USB adapter (802.11n) and integrated network in the clinical facility. In principle, at this early development stage, the results are available to review via any modern based web browser. In our experiment an iPhone 6 (iOS v9.0) was used as an example of a handheld platform to graphically represent and interpret the gait data post walking, Fig. 1.

For transparency, the raw accelerometer data from the wireless wearable (WAX9) was simultaneously streamed and gathered at a computer (Windows ${ }^{\circledR}$ operating system, Bluetooth Belkin USB Adapter v4.0, MATLAB ${ }^{\circledR}$ Instrument Control Toolbox) and analysed with the original (same) MATLAB ${ }^{\circledR}$ based algorithms [9] as those translated into Python ${ }^{\mathrm{TM}}$.

\section{Equipment - validated reference}

The computer equipped with $\mathrm{MATLAB}^{\circledR}$ algorithms, was also used to program a reference (validated) wearable and download its data for post processing using the same gait analysis algorithms deployed on the streamed data [9]. The participant wore the reference wearable (AX3, Axivity, York UK, single tri-axial accelerometer, $100 \mathrm{~Hz}, \pm 8 \mathrm{~g}, 14 \mathrm{bit}$ ) as close as possible to L5 (wearables were placed adjacent to each other). This more labour intensive method ( $\sim 5 \mathrm{mins}$ ) has been previously validated [9] and used here for comparison to the proposed system. This wearable was attached with the methods previously described.

\section{Condition 1: supervised gait assessment}

Typically, a supervised gait assessment involves a trained clinician or researcher monitoring the participant during a number of scripted walking trials, ranging from short intermittent walks (where data is later pooled) and/or a continuous 2 minute walk [18]. The system described herein facilitates gait assessment in any generic clinic or free-living environment. The latter performed in habitual surroundings are thought to be more ecologically valid, reduces burden on outpatient departments and any logistical or financial costs incurred by the participant. (Alternate conditions are presented in the Discussion for future implementation).

\section{Testing scenario A: clinic}

A single participant was used to test the proposed system (M, 32 years, $1.8 \mathrm{~m})$. Two types of gait assessment are usually performed, (i) four repeated intermittent walks and (ii) a 2 minute continuous walk [18]. For the purposes of this pilot study, intermittent walks only were used. The participant walked $12 \mathrm{~m}$ in a straight line. The participant was told to initiate walking with their right foot during each of the four walking trials. This was repeated 4 times. Left/right step detection was estimated by the proposed system (and streamed data) by the polarity of the filtered $\left(4^{\text {th }}\right.$ order, Butterworth, $\left.2 \mathrm{~Hz}\right)$ vertical angular velocity during the detection of an IC [12]. Upon completion of each walk the participant remained still before turning and commencing the next walk. Steps and gait characteristics for the proposed system were computed on the docking unit $\left(\mathrm{Pi} /\right.$ Python $\left.^{\mathrm{TM}}\right)$ and compared to the computer $\left(\right.$ MATLAB $\left.^{\circledR}\right)$ and reference as a cross comparison.

\section{Results}

All left and right steps were accurately detected by the proposed system in comparison to the MATLAB ${ }^{\circledR}$ algorithms. Gait characteristics from the pooled intermittent walks are presented in Table I. The proposed Python ${ }^{\mathrm{TM}}$ based system resulted in values similar to those generated through streamed and logged data analysed by validated MATLAB ${ }^{\circledR}$ algorithms. The proposed system gathered, computed and presented characteristics (similar to that in Fig. 2) in $~ 10$ s compared to the manual downloading and analysis procedures ( $\sim$ mins).

TABLE I. POOLED INTERMITTENT WALKS FROM THE CLINIC

\begin{tabular}{|l|c|c|c|}
\hline Characteristic & Proposed & Streamed & Reference \\
\hline \multicolumn{4}{|c|}{ Mean gait characteristics } \\
\hline Step time (s) & 0.585 & 0.592 & 0.583 \\
Stance time (s) & 0.743 & 0.774 & 0.745 \\
Swing time (s) & 0.428 & 0.415 & 0.413 \\
Step length (m) & 0.712 & 0.701 & 0.667 \\
Step velocity (m/s) & 1.219 & 1.113 & 1.145 \\
\hline \multicolumn{3}{|c|}{ Variability (var.) gait characteristics } \\
\hline Step time var. (s) & 0.039 & 0.039 & 0.031 \\
Stance time var. (s) & 0.045 & 0.058 & 0.029 \\
Swing time var. (s) & 0.024 & 0.028 & 0.023 \\
Step length var. (m) & 0.035 & 0.048 & 0.058 \\
Step velocity var. (m/s) & 0.077 & 0.076 & 0.070 \\
\hline \multicolumn{4}{|c|}{ Asymmetry (asy.) gait characteristics } \\
\hline Step time asy. (s) & 0.018 & 0.018 & 0.014 \\
Stance time asy. (s) & 0.028 & 0.028 & 0.011 \\
Swing time asy. (s) & 0.026 & 0.026 & 0.028 \\
Step length asy. (m) & 0.017 & 0.040 & 0.062 \\
\hline
\end{tabular}




\section{Discussion}

The purpose of this pilot study was to develop and test the feasibility of a portable gait analysis system suitable at low cost. It was important to select components and technologies with no license restriction such that extension, cost and availability do not restrict wide scale system deployment. The proposed system offers a cost-effective alternative to most commercial wearable systems while presenting validated gait characteristics from a clinical conceptual model of gait.

The systems performance capability was compared using an accepted validated analysis approach implemented on a research grade software tool-chain. The test demonstrated that despite the low-cost components, the precision of the tool does not seem to greatly alter. However, a stringent statistical analysis needs to be performed on a larger cohort and within pathology to determine the intra person variability and clinical acceptance. Usability of the tool (automated) was significantly easier and quicker than the traditional manual approach and thus holds promise for design of a more deployable prototype, i.e. no scripting or algorithm knowledge required.

In performing the translation of current $\mathrm{MATLAB}^{\circledR}$ based scripts it was necessary to re-implement functionality stemming from the Signal Processing Toolbox, more specifically the Gaussian continuous wavelet transform (CWT). The methodology is used to detect the IC/FC events within the gait cycle, but had never been previously implemented for Python ${ }^{\mathrm{TM}}$. Both languages feature a standard floating point representation (double precision). However, implementation differences on recursive functions lead to accumulated error. To demonstrate this, consider the following code implemented in $\mathrm{MATLAB}^{\circledR}$ :

$$
\begin{aligned}
& >A=0.8-0.7 ; \\
& >>B=0.1 ; \\
& >>A==B \\
& \text { ans }=0 \\
& >>A-B \\
& \text { ans }=8.32667268468867 e-17
\end{aligned}
$$

In this single, subtractive maths operation, a small error is accumulated. During recursive operations (such as those in the CWT) it is the subtle implementation differences that when combined with rounding errors are thought to have contributed to the precision disagreements observed in Table I as well as the difference in sampling frequencies between wearables. However, determining if these differences are clinically significant warrants further investigation. Moreover this raises technical considerations when contrasting values between systems and algorithm software.

\section{The next step: future work}

A number of computational components will be completed to enhance the usability of the system. These include (i) the transfer of results to a handheld device via Bluetooth to account for lack of any Wi-Fi connectivity and (ii) implementation of macro gait characteristics, to examine behaviour. The latter is only possible during prolonged ( 7 day) monitoring and equates to additional testing scenarios and conditions:

\section{Testing scenario B: clinic/laboratory}

To robustly test the utility of the system on older adults it will be deployed during a range of gait tasks including 2 min continuous walking. This includes use on older adult and neurological cohorts (e.g. Incidence of Cognitive Impairment in Cohorts with Longitudinal Evaluation GAIT project [19]). The pragmatic clinical deployment of the system will be tested in comparison to current methods.

\section{Testing scenario $C$ : free-living (home and community)}

The small size and light weight of the system enables home assessment and the use of intermittent walks in any living environment, Fig. 2. Similar to clinical testing, data can be captured by the docking unit (Python ${ }^{\mathrm{TM}}$ ) and will be relayed via $\mathrm{Wi}-\mathrm{Fi}$ or Bluetooth connectivity. If the researcher deems more in depth patient assessment is necessary, then the wearable can remain worn and the docking unit left in situ within the home (Condition II). This may be to investigate the habitual micro characteristics of the patient, eliminating any observer effect [20] and/or to learn the habitual macro characteristics (e.g. total time walked) during 7 days, understanding any interventional impact of increased physical activity recommendations. 


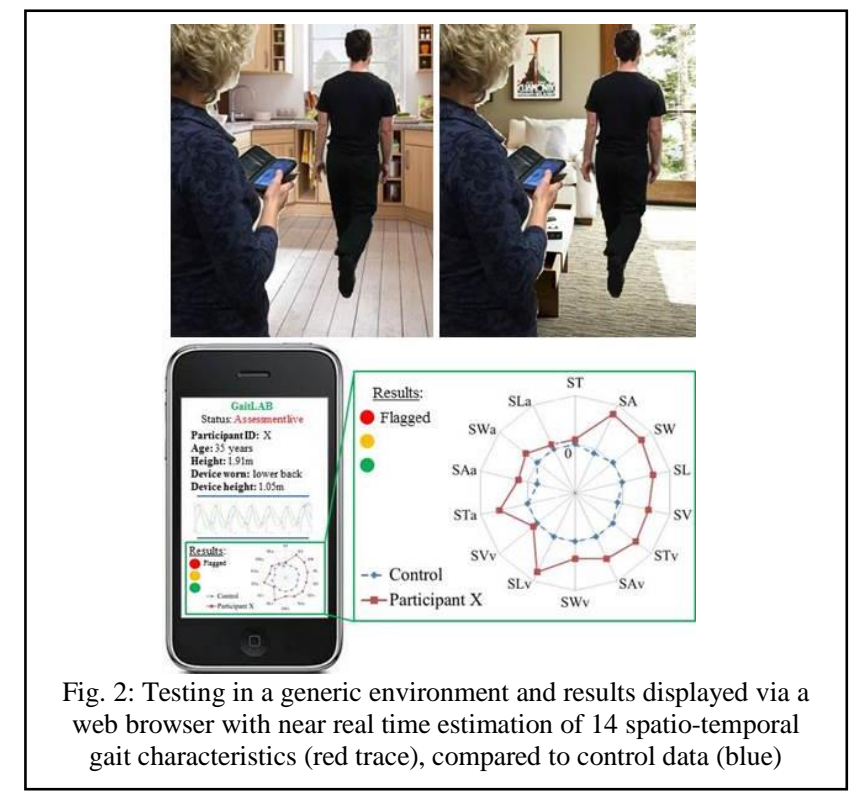

Condition II: unsupervised (longitudinal) gait assessment

During this condition the researcher leaves the docking unit in the participant's home after the initial tests have finished while the participant continues to wear the wearable during waking hours. The dock can be connected to the electrical mains but remain operationally independent for a period of 8 hours due to an internal battery (12000mAh rechargeable lithiumpolymer).

The wearable is equipped to stream data when in close proximity (up to $20 \mathrm{~m}$ direct line of sight). However, when out of range or transmission is obstructed (for example due to internal room boundaries) data is stored on its internal memory. Once within range transmission is automatically restored and data is streamed to the dock.

During nocturnal hours the participant would removes the wearable and place it within its slot on the dock, Fig. 1. This facilitates re-charging. Battery life when sampling gyroscope and streaming over Bluetooth is $\sim 12$ hours. In our anticipated use case, the data is downloaded upon docking, processed and assessed for possible bouts of gait. This is achieved by the processes listed previously, estimating micro and macro levels of gait. This will provide the clinician or researcher with spatio-temporal clinical characteristics of gait in habitual settings and the behavioural patterns of ones ambulatory activity.

\section{Conclusion}

This pilot work demonstrates the feasibility of low-cost open source components to instrument gait, but full (clinical) validity is yet to be determined. Results are encouraging to develop the system into an economical and pragmatic gait analysis tool to deliver a conceptual model of gait incorporating clinical relevant (micro) characteristics. Further developments will involve a robust clinical evaluation and implementation of behavioural outcomes (macro) for a complete gait analysis system during remote (free-living) monitoring.

\section{References}

Lord, S., et al., Independent domains of gait in older adults and associated motor and nonmotor attributes: validation of a factor analysis approach. J Gerontol A Biol Sci Med Sci, 2013. 68(7): p. 820-7.

Verghese, J., et al., Quantitative gait dysfunction and risk of cognitive decline and dementia. J Neurol Neurosurg Psychiatry, 2007. 78(9): p. 92935 .

Beauchet, O., et al., Stops walking when talking: a predictor of falls in older adults? European Journal of Neurology, 2009. 16(7): p. 786-795.

Studenski, S., et al., Gait speed and survival in older adults. JAMA, 2011. 305(1): p. 50-8.

Lara, J., et al., Towards measurement of the Healthy Ageing Phenotype in lifestyle-based intervention studies. Maturitas, 2013. 76(2): p. 189-99.

Godfrey, A., et al., iCap: Instrumented assessment of physical capability. Maturitas, 2015.

Godfrey, A., et al., Direct measurement of human movement by accelerometry. Med Eng Phys, 2008. 30(10): p. 1364-86.

Innerd, P., et al., A comparison of subjective and objective measures of physical activity from the Newcastle 85+ study. Age and Ageing, 2015.

Del Din, S., A. Godfrey, and L. Rochester, Validation of an accelerometer to quantify a comprehensive battery of gait characteristics in healthy older adults and Parkinson's disease: toward clinical and at home use. IEEE J Biomed Health Inform, 2015.

Lord, S., B. Galna, and L. Rochester, Moving forward on gait measurement: toward a more refined approach. Mov Disord, 2013. 28(11): p. 1534-43.

Godfrey, A., et al., The association between retirement and age on physical activity in older adults. Age Ageing, 2014. 43(3): p. 386-93.

McCamley, J., et al., An enhanced estimate of initial contact and final contact instants of time using lower trunk inertial sensor data. Gait Posture, 2012. 36(2): p. 316-8.

Zijlstra, W. and A.L. Hof, Assessment of spatio-temporal gait parameters from trunk accelerations during human walking. Gait Posture, 2003. 18(2): p. 1-10.

Godfrey, A., et al., Instrumenting gait with an accelerometer: a system and algorithm examination. Med Eng Phys, 2015. 37(4): p. 400-7. Chastin, S.F.M. and M.H. Grant
Posture, 2010. 31(1): p. 82-86. 
16. Lyons, G.M., et al., A description of an accelerometer-based mobility monitoring technique. Med Eng Phys, 2005. $27(6)$ : p. $497-504$.

17. Ladha, C., et al. DEMO: OpenMovement Sensor Platform. in Digital Futures 2012. 2012. Aberdeen, Scotland: RCUK Digital Economy.

18. Galna, B., S. Lord, and L. Rochester, Is gait variability reliable in older adults and Parkinson's disease? Towards an optimal testing protocol. Gait Posture, 2013. 37(4): p. 580-5.

19. Lord, S., et al., Ambulatory activity in incident Parkinson's: more than meets the eye? J Neurol, 2013. 260(12): p. $2964-72$.

20. Robles-Garcia, V., et al., Spatiotemporal Gait Patterns During Overt and Covert Evaluation in Patients With Parkinson s Disease and Healthy Subjects: Is There a Hawthorne Effect? J Appl Biomech, 2015. 31(3): p. 189-94. 